\title{
Spatio-Temporal Patterns with Hyperchaotic Dynamics in Diffusively Coupled Biochemical Oscillators
}

\author{
GEROLD BAIER* and SVEN SAHLE \\ Institute for Plant Biochemistry, University of Tübingen, D-72076 Tübingen, Germany
}

(Received 9 October 1996)

\begin{abstract}
We present three examples how complex spatio-temporal patterns can be linked to hyperchaotic attractors in dynamical systems consisting of nonlinear biochemical oscillators coupled linearly with diffusion terms. The systems involved are: (a) a two-variable oscillator with two consecutive autocatalytic reactions derived from the Lotka-Volterra scheme; (b) a minimal two-variable oscillator with one first-order autocatalytic reaction; (c) a three-variable oscillator with first-order feedback lacking autocatalysis. The dynamics of a finite number of coupled biochemical oscillators may account for complex patterns in compartmentalized living systems like cells or tissue, and may be tested experimentally in coupled microreactors.
\end{abstract}

Keywords: Biochemical oscillations, Spatio-temporal hyperchaos, Complex patterns, Information processing

\section{INTRODUCTION}

Complex biochemical spatio-temporal self-organization with high-dimensional chaotic attractors cannot only occur in continuous reaction-diffusion systems with infinitely many degrees of freedom in phase space. The discovery that linear diffusive coupling of only two oscillatory reaction schemes can produce chaos [1] nurtures the idea that finitely many coupled oscillators can be employed to generate spatio-temporal patterns with underlying attractors of arbitrarily high (finite) dimension in phase space [2]. Here we investigate the concept of attractor dimension increase associated with an increasing number of positive Lyapunov characteristic exponents (LCEs), i.e. hyperchaos, in three examples of coupled biochemical oscillators.

Following the introduction of the prototypic flow with a hyperchaotic attractor we first demonstrate hyperchaos of coupled oscillators in one spatial dimension where the two coupled species have equal diffusion coefficients. The second example illustrates the transition from a stable Turing structure to spatio-temporal hyperchaos, both spatially trapped between two oscillatory

* Corresponding author. 
regimes of differing amplitude and frequency in a parameter gradient. The third example shows the transition from low to high-dimensional hyperchaos in a two-dimensional grid of biochemical oscillators.

\section{ABSTRACT HYPERCHAOS}

To introduce hyperchaos we present a four-variable ordinary differential equation composed of two well-known subsystems. The equation is:

$$
\begin{aligned}
\dot{X}_{1} & =-X_{2}+a_{1} X_{1}, \\
\dot{X}_{2} & =X_{1}-Z, \\
\dot{Y} & =-Z+a_{2} Y, \\
\dot{Z} & =0.1+3 Z\left(b_{1} X_{2}+b_{2} Y\right)-8 Z
\end{aligned}
$$

with $a_{1}, a_{2}, b_{1}, b_{2}>0$.

For $b_{1}=1, b_{2}=0$, the subsystem $\left(X_{1}, X_{2}, Z\right)$ is Rössler's equation for continuous chaos. Parameter $a_{1}$ can be tuned to change the system's stable attractor from a single-loop limit cycle via a period-doubling sequence to a chaotic attractor. The stretching and folding of the flow is realized with the switching nonlinearity in the equation for $Z$ [3]. For $b_{1}=0, b_{2}=1$, the subsystem $(Y, Z)$ forms an attracting limit cycle when $a_{2}$ exceeds the value of the Hopf bifurcation. As this limit cycle works with the same nonlinearity as the first subsystem, one can view such a structure as the oscillatory source of the chaotic attractor. Combining the two subsystems $\left(b_{1}=1, b_{2}=1\right)$, as in Eq. (1), yields a hyperchaotic flow: the harmonic oscillator of the chaotic subsystem oscillates in the $\left(X_{1}, X_{2}\right)$ plane. Both subsystems keep expanding for small values of variable $Z$ due to nonzero expansion parameters $a_{1}$ and $a_{2}$, respectively. The two switching nonlinearities in $\mathrm{d} Z / \mathrm{d} t$ then increase the values of variables $\mathrm{X}_{2}$ and $Y$, and return the value of $Z$ sharply close to zero whenever the threshold is passed. We thus have an explicit implementation of the principle of an oscillator expanding in two directions in phase space together with the flow being folded back in two orthogonal directions. The numerical calculation of the spectrum of Lyapunov exponents yields two positive LCEs. A Poincaré cross section of a numerically simulated hyperchaotic attractor of Eq. (1) is shown in Fig. 1

Equation (1) solves the mystery of Rössler's first equation for hyperchaos [4]. That pioneering system possessed a hyperchaotic attractor for special choices of parameters and initial conditions, most choices in the neighborhood, however, lead to an escape of the trajectory to either fixed point or infinity. Comparing the two equations it turns out that only the coupling of expanding variable $Y$ to the chaotic subsystem is different. Coupling the two linear subsystems equivalently to $Z$ (as in Eq. (1)) yields a hyperchaotic flow that is prototypic in the same sense as the subsystem $\left(X_{1}, X_{2}, Z\right)$ is prototypic for chaotic flows. Incidentally, the system of Eq. (1) can be transformed into the simplest hyperchaotic flow [5] (containing only one nonlinearity) by means of a mode transformation of the linear variables [6]. Thus, the extensions to chaos with even more than two directions of mean divergence work naturally as in [5].

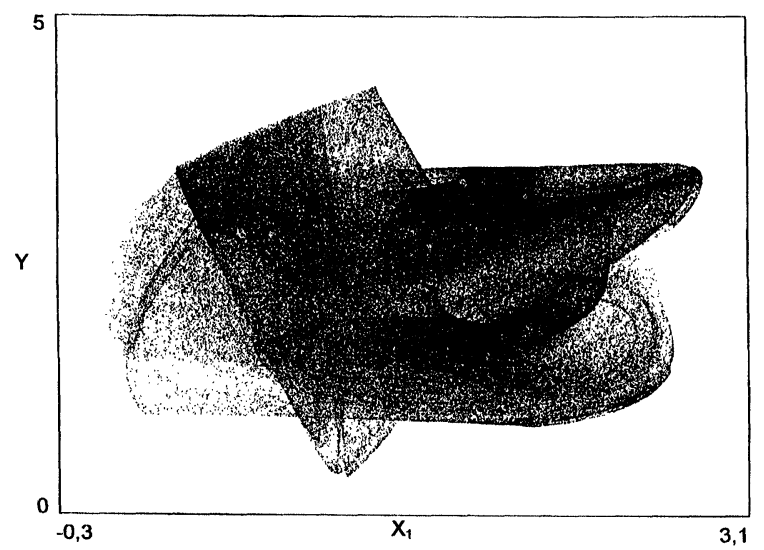

FIGURE 1 Poincaré cross-section of hyperchaotic flow in Eq. (1) taken at $x_{2}=0$ with $a_{1}=0.0, a_{2}=0.3, b_{1}=b_{2}=1$. 


\section{SPATIO-TEMPORAL HYPERCHAOS WITH TWO EQUAL DIFFUSION COEFFICIENTS}

We now describe the occurrence of spatio-temporal hyperchaos in diffusively coupled (bio)chemical oscillators. The first oscillator employed is a chemical realization of the $(Y, Z)$ limit cycle subsystem $\left(b_{1}=0\right)$ of Eq. (1). The rate equations are:

$$
\begin{aligned}
\dot{X} & =0.01+b X(Y-d), \\
\dot{Y} & =Y-b X Y+a Y^{2}
\end{aligned}
$$

with $a, b, d>0$.

The mechanism consists of two consecutive autocatalytic steps in variables $X$ and $Y$, a zeroorder input to $X$ and a first-order decay of variable $X$. This Lotka-Volterra scheme is extended by the instability-inducing second-order autocatalysis governed by parameter $a$. Homogeneous oscillating units with the kinetics of Eq. (2) can now be coupled linearly by diffusive terms for both variables as follows:

$$
\begin{aligned}
\dot{X}_{1} & =f_{x}+D_{x}\left(X_{2}-X_{1}\right), \\
\dot{Y}_{1} & =f_{y}+D_{y}\left(Y_{2}-Y_{1}\right), \\
\dot{X}_{i} & =f_{x}+D_{x}\left(X_{i+1}-2 X_{i}+X_{i-1}\right), \\
\dot{Y}_{i} & =f_{y}+D_{y}\left(Y_{i+1}-2 Y_{i}+Y_{i-1}\right), \\
\dot{X}_{N} & =f_{x}+D_{x}\left(X_{N-1}-X_{N}\right), \\
\dot{Y}_{N} & =f_{y}+D_{y}\left(Y_{N-1}-Y_{N}\right)
\end{aligned}
$$

with $i=2,3, \ldots,(N-1) . f_{x}$ and $f_{y}$ denote kinetic terms, e.g. as in Eq. (2); $D_{x}$ and $D_{y}$ are the respective diffusion coefficients of variables $X$ and $Y$; and $N$ is the number of cells. The scheme is written with zero-flux boundary conditions, i.e. for an open chain of reaction cells. However, the results presented can qualitatively be achieved with periodic boundary conditions as well.

Previous results on hyperchaos with a finite number of diffusively coupled cells were obtained with a ratio of diffusion coefficients considerably smaller or larger than one (see e.g. [2,7]). In con-

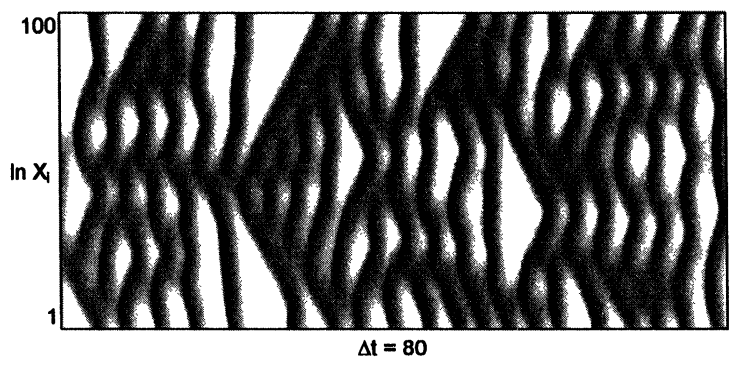

FIGURE 2 Spatio-temporal evolution of 100 oscillators Eq (2) coupled in the form of Eq. (3) with $a=1, b=3, d=1$, $D_{x}=D_{y}=5$. Logarithms of the concentrations of variables $X_{i}$ were grey coded from -4 (white) to 2 (black)

trast, we now couple five cells of the oscillator in Eq. (2) in the oscillatory parameter domain with equal diffusion coefficients, i.e. a ratio $D_{x} / D_{y}=1$. For a coupling strength of $D_{x}=D_{y}=0.05$ we observe a chaotic attractor with the four largest LCEs $(0.2,0.1,0,-0.3)$. The attractor is hyperchaotic with two positive LCEs. Under otherwise identical parameters the hyperchaotic attractor also exists for a ratio of diffusion coefficients slightly smaller or slightly larger than one. Increasing the number of coupled cells leads to hyperchaotic attractors with an increasing number of positive LCEs for a finite window of coupling parameters $D_{x}=D_{y}$. Figure 2 is a spacetime plot for 100 coupled cells in the hyperchaotic regime. Again the hyperchaos exists for ratios $D_{x} / D_{y}$ slightly smaller and slightly larger than one. Thus, in addition to the cases $D_{x}>D_{y}$ and $D_{x}<D_{y}$, spatio-temporal hyperchaos has been found to exist generically for the case $D_{x} / D_{y}=1$.

\section{TURING PATTERN AND HYPERCHAOS TRAPPED BETWEEN TWO LIMIT CYCLES}

The simplest biochemical oscillator with two variables contains only one first-order autocatalysis:

$$
\begin{aligned}
\dot{X} & =a+X Y-c_{1} X /\left(c_{2}+X\right), \\
\dot{Y} & =b-X Y
\end{aligned}
$$


with $a, b, c_{1}, c_{2}>0$. This system has been found to produce spatio-temporal hyperchaos for $D_{x}>D_{y}$ [2] and for $D_{x}<D_{y}$ [8] in the arrangement of Eq. (3). In the latter case, i.e. under Turing conditions, the hyperchaos is a chaotic mixing of unstable Hopf and Turing modes. We have studied the dynamics of diffusively coupled oscillators Eq. (4) in a gradient of parameter $a$ :

$$
\begin{aligned}
\dot{X}_{1}= & f_{x}+D_{x}\left(X_{2}-X_{1}\right), \\
\dot{Y}_{1}= & f_{y}+D_{y}\left(Y_{2}-Y_{1}\right), \\
\dot{X}_{i}= & f_{x}-(i-1) \delta /(N-1) \\
& +D_{x}\left(X_{i+1}-2 X_{i}+X_{i-1}\right), \\
\dot{Y}_{i}= & f_{y}+D_{y}\left(Y_{i+1}-2 Y_{i}+Y_{i-1}\right), \\
\dot{X}_{N}= & f_{x}-\delta+D_{x}\left(X_{N-1}-X_{N}\right), \\
\dot{Y}_{N}= & f_{y}+D_{y}\left(Y_{N-1}-Y_{N}\right)
\end{aligned}
$$

with $i=2,3, \ldots,(N-1)$.

The gradient in the input to variable $X$ was chosen such that the first cell of the decoupled system oscillates in a small-amplitude sinusoidal fashion and the last oscillator possesses a largeamplitude relaxational limit cycle with lower frequency (parameters are given in the caption of Fig. 3). For strong coupling under Turing conditions $\left(D_{x}<D_{y}\right)$ the system Eq. (5) with kinetic terms taken from Eq. (4) organizes itself into a stable asymmetric Turing pattern, e.g. for $N=100$. Decreasing the coupling strength leads to the appearance of oscillating behavior at both spatial ends of the system. Figure 3(a) is a space-time plot of this situation. A stationary Turing pattern is located in the central area. It is hemmed in by oscillatory cells. Interestingly, the oscillations are related to those of the uncoupled system and thus differ from each other in amplitude and frequency. The Turing structure dynamically separates the two oscillatory domains. This is a remarkable situation as it seems impossible to extract information about the distant oscillator from a time series of one oscillator if only finite precision is available, e.g. as provided by experimental data. Thus, with a moderate parameter
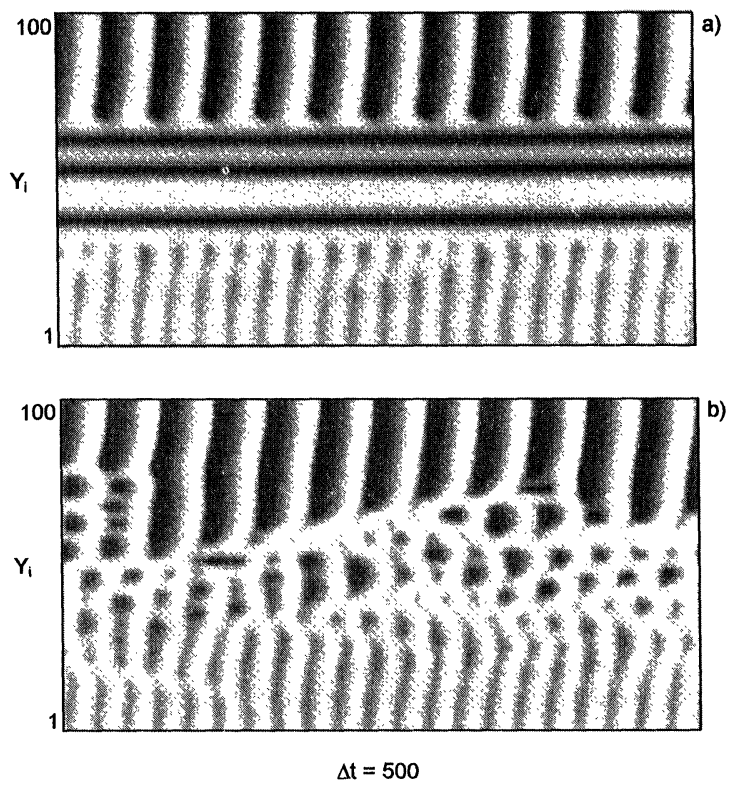

FIGURE 3 Spatio-temporal evolution of 100 oscillators Eq. (4) coupled in the form of Eq. (5) with $a=0.057, b=0.12$, $c_{1}=0.5, c_{2}=0.1, \delta=0.01$. Logarithms of the concentrations of variables $Y_{i}$ were grey coded. (a) $D_{x}=0.07, D_{y}=0.4$, (b) $D_{x}=0.055, D_{y}=0.2$.

gradient, diffusive coupling results in dynamic decoupling of otherwise identical oscillators.

For weaker coupling the Turing structure breaks up and makes way for a ribbon of chaotic behavior caught between the two oscillatory hems (Fig. 3(b)). Again the situation is such that if an attractor reconstruction would be tried from a single time series the astonished researcher could find three qualitatively different results (sinusoidal periodic, chaotic, relaxational periodic) depending on which time series she or he would use, even though the system has settled to a single well-defined attractor. Figure 4 shows two phase space projections of the trajectory. Variables $X_{1}$ and $X_{98}$ (left side) fill a rectangular plane as if they were two independent periodic oscillators, whereas variables $X_{51}$ and $X_{53}$ (right side) form a familiar chaotic attractor as in the case of hyperchaos in diffusively coupled cells under Turing conditions [9]. Due to the close relationship with the hyperchaos in [8] we suspect that the chaotic region of Fig. 3(b) possesses 

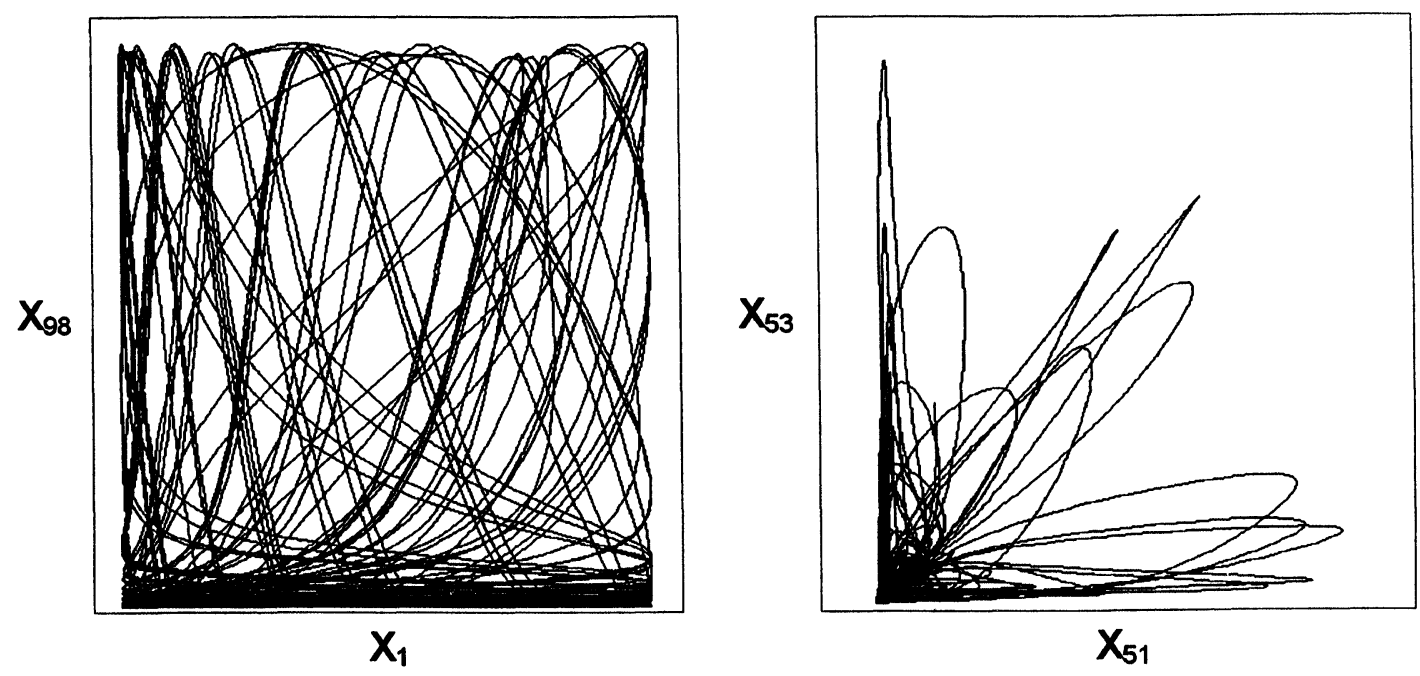

FIGURE 4 Phase space projection of two variables for the attractor in Fig. 3(b). Left: One variable from the high and low frequency oscillation, respectively. Right: Two variables from the chaotic region.

more than one positive LCE but a reliable LCE estimation of the two-hundred dimensional system has yet to be performed.

\section{HYPERCHAOTIC PATTERNS IN TWO SPATIAL DIMENSIONS}

In our final example we employ Thron's oscillator [10] which lacks autocatalysis and instead creates the oscillatory instability of a fixed point by feedback inhibition in a three-variable scheme:

$$
\begin{aligned}
& \dot{X}=a /\left(k_{m}+Z\right)-X, \\
& \dot{Y}=X-Y, \\
& \dot{Z}=Y-k_{1} Z /\left(k_{m}+Z\right)
\end{aligned}
$$

with $a, k_{1}, k_{m}>0$.

We have recently found that diffusive coupling of three such cells creates a hyperchaotic attractor [11]. Now we go further and couple three times three cells on a square grid with nextneighbor couplings of each cell. For simplicity, periodic boundary conditions (toroidal topology) is chosen but, as in Section 3, the qualitative result can also be obtained with zero-flux boundaries. Calculating the spectrum of Lyapunov exponents for the set of parameters: $a=0.1$, $k_{1}=0.48, k_{m}=0.001$ with coupling in variable $Z$ $\left(D_{z}=0.005\right.$, and $\left.D_{x}=D_{y}=0\right)$ we find four positive LCEs. For the same set of parameters we also observe hyperchaos on a toroidal grid composed of $10 \times 10$ oscillators Eq. (6). Figure 5(a) is a simulation of this system. The decay of correlation between neighboring cells due to the hyperchaos is reflected in the noncoherent irregular pattern of grey values.

In order to visualize the spatio-temporal pattern associated with a hyperchaotic attractor in more detail we keep the length of the system in Fig. 5(a) constant but cover the area with a grid of $100 \times 100$ oscillators with properly adjusted diffusion coefficient $D_{z}$. Figure 5(b) shows an aperiodic patterns at one instant during the simulation. The pattern is nonstationary and modulated by chaotic oscillations in space and time. A full account of its structural richness necessarily has to include the temporal dimension. However, the strategy to find the 2D spatio-temporally hyperchaotic pattern starting from the dynamics of only a few coupled cells worked reliably as in the 1D cases. 

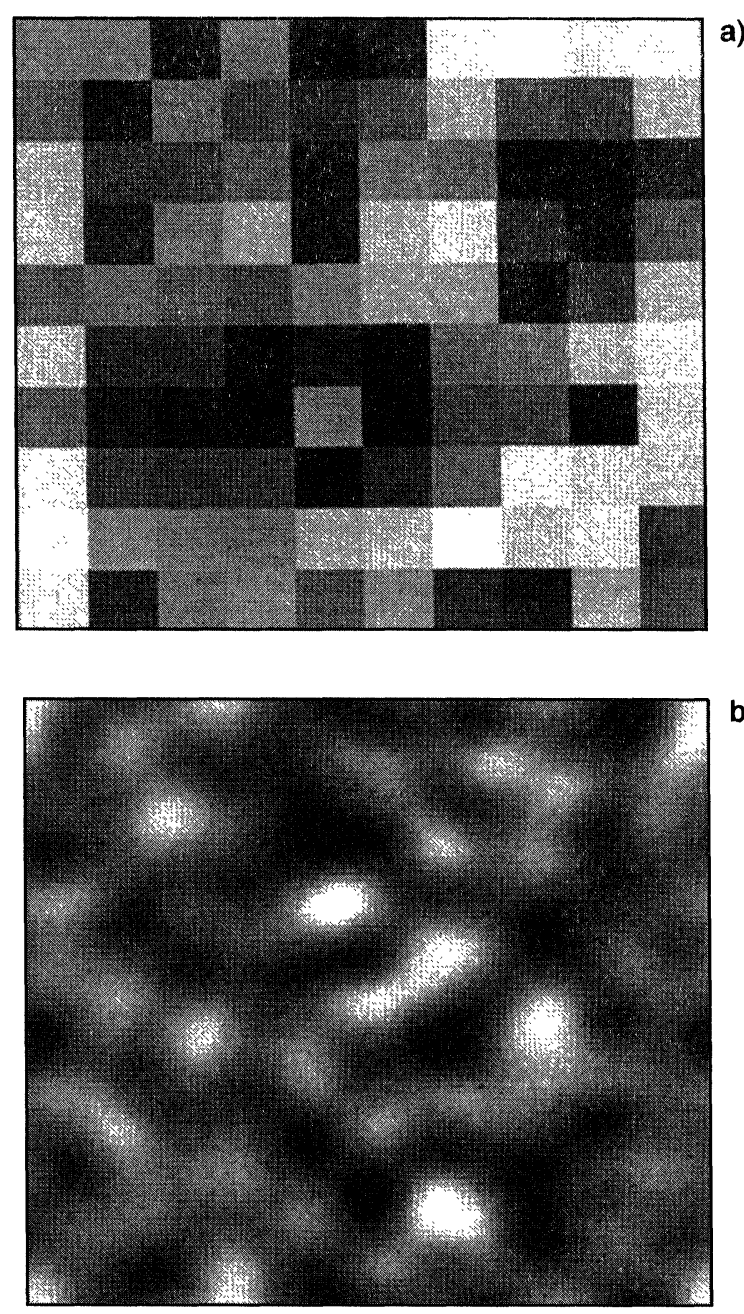

FIGURE 5 Two-dimensional grid of oscillators Eq. (6) with $a=0.1, k_{M}=0.001, k_{1}=0.48$. Each cell is diffusively coupled to its four next neighbors and the grid has periodic boundary conditions. Concentrations of variables $Z_{i j}$ were grey coded. (a) $10 \times 10$ cells, $D_{x}=D_{y}=0, D_{z}=0.005$, (b) $100 \times 100$ cells, $D_{x}=D_{y}=0, D_{z}=0.5$.

\section{DISCUSSION}

Spatio-temporally hyperchaotic patterns are a generic feature of diffusively coupled biochemical oscillators. In particular, the case where two coupled oscillators possess a chaotic attractor can be exploited to generate hyperchaos in a linear chain or in a closed ring of oscillators. Just as hyperchaos can be created out of ordinary chaos by adding new variables with proper coupling as in Eq. (1), the dimension and the number of positive LCEs can be increased stepwise if the number of spatially coupled oscillatory cells is increased. In addition to the case where the diffusion coefficients of two oscillator variables differ by one magnitude, an explicit example could be found to demonstrate spatio-temporal hyperchaos in a system with two equal diffusion coefficients. Thus no principal requirements are imposed beforehand on the ratios of diffusion coefficients in experimental systems.

In systems with one spatial dimension hyperchaotic patterns have now been observed in biochemical models of kinetic oscillations with second-order autocatalysis, as in Eq. (2), firstorder autocatalysis, as in Eq. (4), or in a model with no autocatalysis at all, Eq. (6). The important feature of the oscillators is not necessarily their kinetic structure. For instance, equivalent hyperchaotic dynamics were described for two different autocatalytic models, one an activatorinhibitor system, the other a substrate--depletion system [8]. The trick is to find a set of kinetic parameters which lead to at least two positive LCEs under diffusive coupling of a small number of cells (Eq. (3)). These parameters can then be used for the higher-dimensional cases with the coupling strength (diffusion coefficients) as bifurcation parameter.

A new direction of investigation will now be to study the dynamics of nonidentical coupled cells. The obvious motivation for this is that biological or biochemical systems in general comprise a variety of cells with a distribution of kinetic parameters or coupling constants. A first step in that direction was the study of a one-dimensional chain of oscillators with a gradient of one parameter. In addition to the fact that hyperchaos can survive nonidentical parameters in coupled cells the possibility arises to combine different dynamic substructures in one composite pattern. In one case a nonhomogeneous fixed point (Turing) structure separated two oscillatory events of differing amplitude and frequency ("dynamic wall"), and in 
another case an aperiodic spatio-temporal pattern could be confined to a subset of the system by periodic neighbors (Figs. 2 and 3). Such composite structures may for example occur in experimental systems where the presence of frequency gradients has been established (see e.g. [12]).

Aperiodic patterns on two-dimensional grids indicate that the connection between low-dimensional hyperchaotic attractors and high-dimensional hyperchaotic spatio-temporal patterns can be expected in experimental systems with two spatial extensions, e.g. in thin liquid layers or in a single layer of biological cells. We expect that spatio-temporal patterns with low spatial correlation and rich structure can naturally arise in tissue of living cells with oscillatory characteristics. Recently we have observed spatio-temporal hyperchaos in diffusively coupled kinetic systems proposed for the explanation of cytosolic calcium oscillations by Goldbeter, Dupont and Berridge [13].

An important property of all systems presented is the fact that the high-dimensional patterns were generated in finite-dimensional systems. Directly interpreted the equations model a finite number of (bio)chemical reactors with continuous supply of at least one (bio)chemical species. They can thus easily be extended to describe a set of coupled continuous flow well-stirred tank reactors. To build and run such a set on a macroscopic scale appears tiresome. Yet in the case of enzymatic reactions one could think of a realization as a set of microreactors. In that case the assumption that all cells are identical and that a component is pumped at equal rate through all reactors is just. The technology of microreactors will allow the detailed investigation of high-dimensional chaotic attractors in finitedimensional systems with an accuracy which can never be expected in living systems.

The idea of coupled microreactors with full control of flows and coupling strengths leads us to speculate whether such a system could undergo structure-formation on an even higher level. Suppose the concentrations in each cell could be mea- sured by a sensor and the couplings between cells were adjustable. Then the coupling strength between cells could easily be made a function of the exchange of (bio)chemicals (i.e. information) between cells. The coupling strengths could then be made functions of the dynamics similar to the weights in artificial neural networks. The result would be a chemical network capable of learning to interpret external patterns (provided e.g. in the form of flow rates) in terms of a set of adjusted coupling parameters. At present there seems to be no clue as to whether living systems use this principle of adjusted couplings of compartments to handle information processing in enymatic reaction networks. However, Bray has elaborated how the kinetics of metabolic reactions may relate to "learning" in neural networks [14]. A proper set of coupled microreactors would be an ideal model experiment to test both the complex self-organization and the information processing capabilities of diffusively coupled biochemical reactors.

\section{Acknowledgements}

We thank Peter Plath for inspiration and discussion; Thomas Kirner, Peter Strasser, Otto Rössler and Jack Hudson for discussion; and the Deutsche Forschungsgemeinschaft and the Fonds der Chemischen Industrie for financial support.

\section{References}

[1] O.E. Rössler (1976), Z. Naturforsch. 31a, 1168.

[2] G. Baier, S. Sahle, U. Kummer and R. Brock (1994), Z. Naturforsch. 49a, 835 .

[3] O.E. Rössler (1976), Phys. Lett. A 57, 397.

[4] O.E. Rössler (1979), Phys. Lett. A 71, 155.

[5] G. Baier and S. Sahle (1995), Phys. Rev. E 51, R2712.

[6] M. Bünner and T. Meyer, private communication.

[7] G. Baier, P. Strasser and U. Kummer (1995), Z. Naturforsch. 50a, 1147.

[8] P. Strasser, O.E. Rössler and G. Baier (1996), J. Chem. Phys. 104, 9974.

[9] P. Strasser (1995), diploma thesis, University of Tübingen [in German].

[10] C.D. Thron (1991), Bull. Math. Biol. 53, 383.

[11] G. Baier and S. Sahle (1997), J. Theor. Biol., submitted.

[12] E. Diamant and A. Bortoff (1969), Am. J. Physiol. 216, 301.

[13] G. Baier, J.-P. Chen and A.A. Hoff [unpublished results].

[14] D. Bray (1990), J. Theor. Biol. 143, 215. 


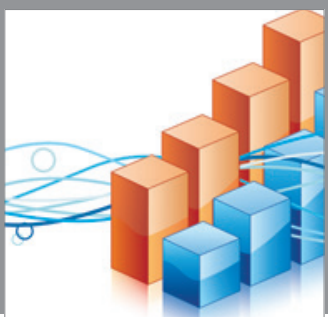

Advances in

Operations Research

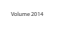

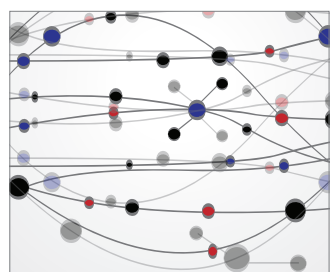

\section{The Scientific} World Journal
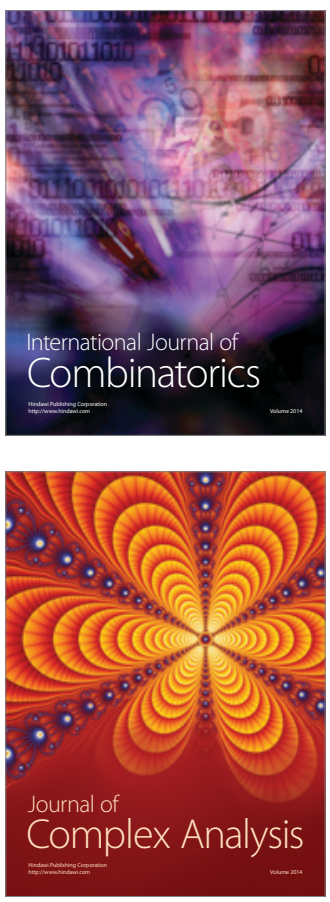

International Journal of

Mathematics and

Mathematical

Sciences
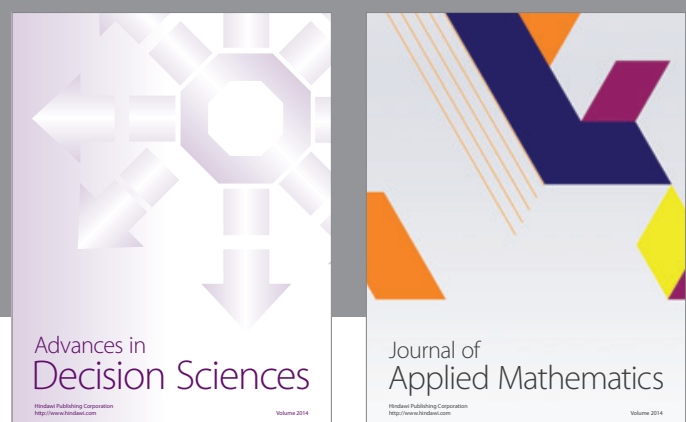

Journal of

Applied Mathematics
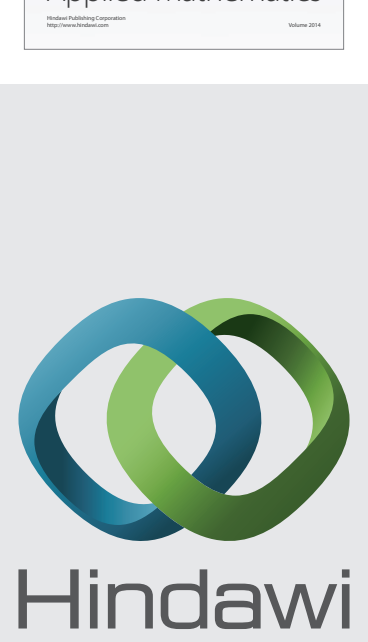

Submit your manuscripts at http://www.hindawi.com
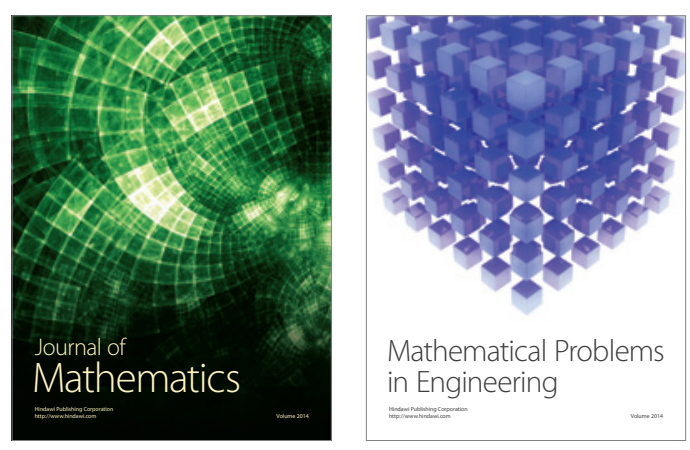

Mathematical Problems in Engineering
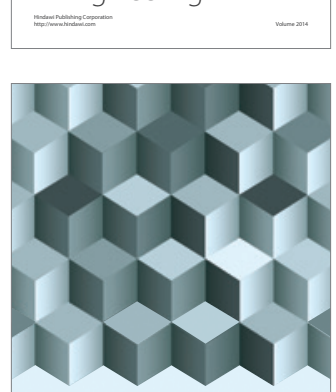

Journal of

Function Spaces
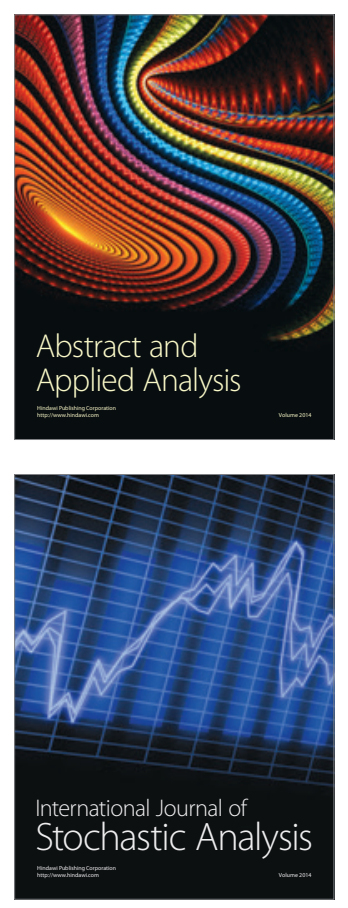

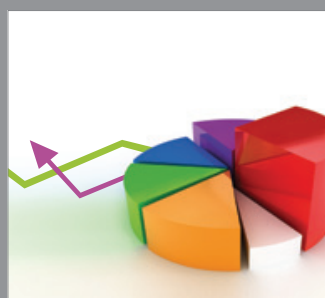

ournal of

Probability and Statistics

Promensencen
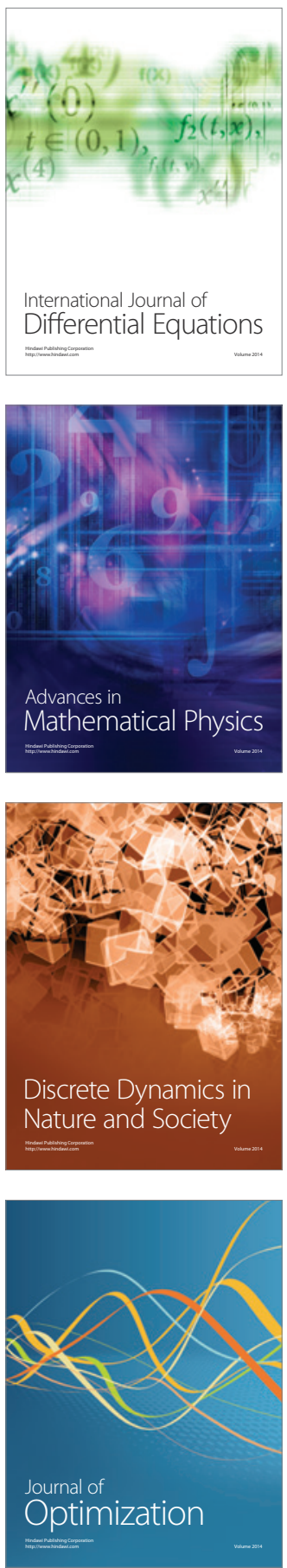